\title{
A Novel Three-State Contactless RF Micromachined Switch for Wireless Applications
}

\author{
J. Pal, Y. Zhu, Senior Member, IEEE, J. Lu, Senior Member, IEEE, F. Khan, and D. Dao
}

\begin{abstract}
This letter presents a novel three-state contactless radio frequency $(\mathrm{RF})$ microelectromechanical systems switch for wireless applications. The switch is free from stiction and charge injection issues occurred inherently in contact-type RF MEMS switches, thereby increasing reliability and lifetime. The contactless switch is based on variable capacitance between signal lines and movable grounded electrodes controlled by electrostatic actuator. The movable grounded electrode has the capability to move bidirectionally, and therefore, the switch can change among ON-, OFF-, and deep OFF-states. Thus, additional isolation can be achieved in the deep OFF-state. The RF measurement results show that the contactless switch has a capacitance tuning ratio of 5.25 between $\mathrm{OFF}$ - and $\mathrm{ON}$-states, and a higher tuning ratio of 11.18 between deep OFF- and ON-states. In addition, the switch exhibits $-3.62 \mathrm{~dB}$ insertion loss and $-\mathbf{2 4 . 4 3 \mathrm { dB }}$ isolation at $2.4 \mathrm{GHz}$. At $5 \mathrm{GHz}$, the insertion loss and isolation are $-2.95 \mathrm{~dB}$ and $-\mathbf{2 0 . 6 5} \mathrm{dB}$, respectively.
\end{abstract}

Index Terms-RF MEMS switches, contactless switch, three-state switch, SOIMUMPs, tunable capacitor.

\section{INTRODUCTION}

$\mathbf{T}$ HE Microelectromechanical Systems (MEMS) switches have achieved considerable interest because of excellent electrical performance compared to the traditional solid-state solutions, for example, high linearity, high isolation, low insertion loss, small size, and low cost. Currently, MEMS switches are mainly metal-contact type and capacitive coupling type switches. The metal-contact switches are associated with an ohmic contact between two metal electrodes, whereas capacitive coupling switches consist of a thin dielectric film and an air gap between two metal electrodes. The air gap changes with applied bias to achieve a change in capacitance between the OFF state and ON state. However, the long term reliability issues in traditional MEMS devices have limited their use in commercial applications [1]. There are two main issues: 1) micro-welding and stiction problems in the metal contacting switches; and 2) dielectric charging problems due to large contact area of the switch in capacitive coupling switches. In order to solve the problems, a contactless type RF MEMS switch for radar applications has been reported [2].

This work was supported in part by the Griffith University Research Infrastructure Program and in part by the Queensland Node through the Australian National Fabrication Facility

The authors are with the Queensland Micro and Nanotechnology Centre, Griffith University, Nathan, QLD 4111, Australia (e-mail: y.zhu@griffith.edu.au).

Color versions of one or more of the figures in this letter are available online at http://ieeexplore.ieee.org.
The switch is operated at electrical resonance frequency to achieve higher isolation.

In this letter, a novel contactless switch is introduced, which is based on the capacitance change between RF signal lines and movable grounded electrodes controlled by electrostatic actuator. The main advantage of the proposed design is that the RF signal transmission varies between deep isolating state (deep-OFF state) and conducting state (ON state) without physical contact between signal lines and movable electrodes. This is achieved by moving the grounded electrodes bi-directionally with high resolution of analog tuning between minimum and maximum capacitances, which offers the benefit of extended tuning range. In addition, the actuators are embedded inside the coplanar waveguide (CPW), thereby reducing any discontinuity in the CPW [3]. In contrast to many electrostatically actuated MEMS switches, the DC biasing is separated from the RF signal path. The proposed switch does not require any ohmic contact or dielectric layer that is liable to stiction or dielectric-charging, therefore the reliability of the device is improved.

The proposed device is designed for wireless applications, for example, dual band antenna with $2.4 \mathrm{GHz}$ and $5 \mathrm{GHz}$ frequencies. The device is fabricated using a commercially available low-cost SOIMUMPS process, which offers a through-hole structure that greatly reduces substrate loss to improve RF performances. The isolation is further improved by actuating the device into deep-OFF state [4]. The measurement results show large capacitance tuning ratio and superior RF performances.

\section{DESIGN AND FABRICATION}

A schematic diagram of the proposed design is shown in Fig 1. The device can change the capacitance between signal lines and laterally movable grounded electrodes in a coplanar waveguide (CPW). In the OFF state, the capacitance between the fixed and movable electrodes is small enough to block most RF signal by the large impedance at $2.4 \mathrm{GHz}$ and $5 \mathrm{GHz}$. When an actuation voltage $\left(\mathrm{V}_{1}\right)$ is applied across the two capacitive actuation electrodes, the laterally movable grounded electrodes are brought closer to the signal lines, therefore large capacitance and low impedance can be achieved to allow RF signal to pass through. As a small air gap $(0.5 \mu \mathrm{m})$ exists between the signal lines and movable grounded electrodes in $\mathrm{ON}$ state, the main barrier limiting the reliability of contact type MEMS switches can be removed.

To optimize the device design and investigate the RF performance, the finite integration technology has been utilized 


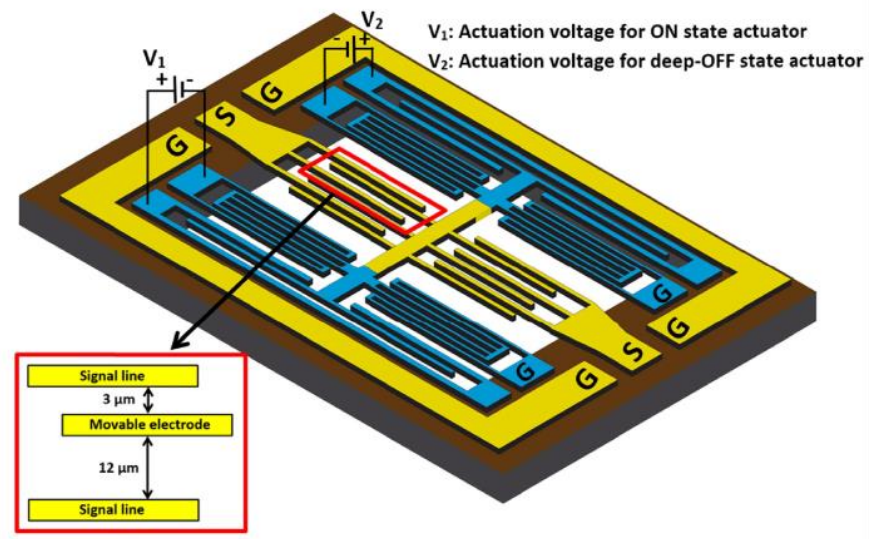

Fig. 1. Schematic diagram of the proposed design. The inset shows the initial gap between the signal lines and grounded movable electrodes.

TABLE I

Key Dimension Parameters of the Fabricated Device

\begin{tabular}{c|c}
\hline Parameters & Value $(\mu \mathrm{m})$ \\
\hline Gaps between signal line and movable electrode & $3 \& 12$ \\
Overlap of signal line and movable electrode & 400 \\
Gap between two actuation electrodes & 10 \\
Width of signal line and movable electrode & 20 \\
Thickness of device & 25 \\
\hline
\end{tabular}

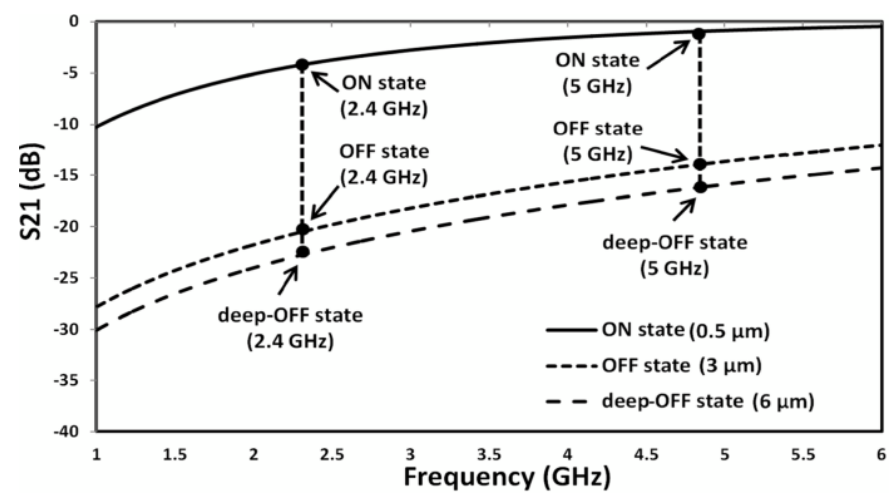

Fig. 2. Simulated S21 parameter results of the proposed design.

on the proposed design by CST Microwave Studio. The key dimension parameters of the device are summarized in Table I. In the OFF state, the movable grounded electrodes are far away $(3 \mu \mathrm{m})$ from the fixed conductors and the most signal is blocked at $2.4 \mathrm{GHz}$ and $5 \mathrm{GHz}$. The continuous analog tuning is achieved by displacing the movable grounded electrodes by applying a DC voltage between the fixed and movable electrode as shown in Fig 1. The pull-in effect will not happen as the full displacement required for tuning is less than $3 \mu \mathrm{m}$, which is smaller than $1 / 3$ of the actuator's gap of $10 \mu \mathrm{m}$. The finite integration simulation results of the designed switch are shown in Fig 2. In the OFF state ( $3 \mu \mathrm{m}$ air gap), the isolations of $-20.15 \mathrm{~dB}$ at $2.4 \mathrm{GHz}$ and $-13.81 \mathrm{~dB}$ at $5 \mathrm{GHz}$ are achieved. When the air gap of the switch is changed from $3 \mu \mathrm{m}$ to $0.5 \mu \mathrm{m}$, i.e. switching from OFF state to

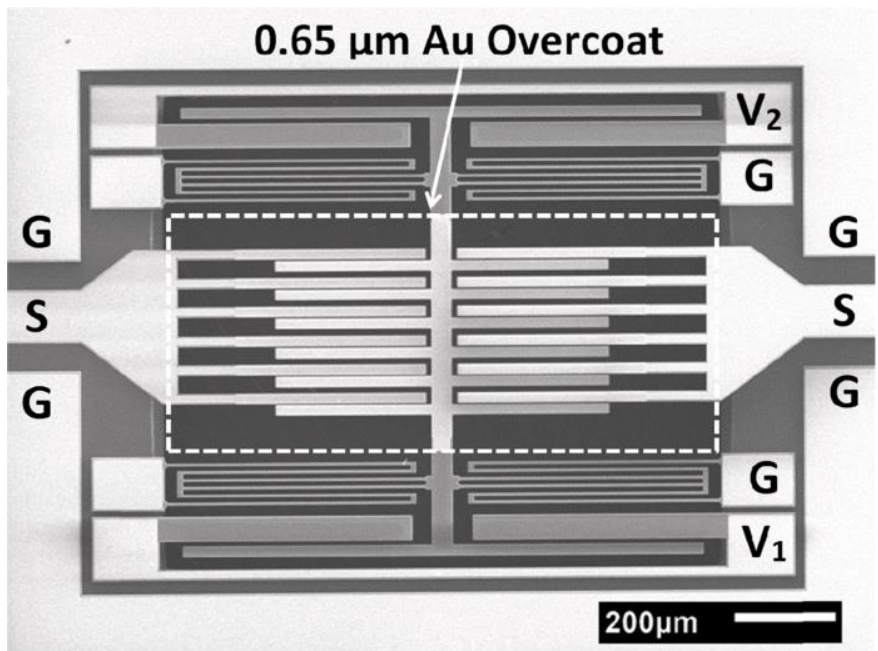

Fig. 3. SEM image of the fabricated micro-switch.

ON state, the insertion losses are $-3.98 \mathrm{~dB}$ and $-0.86 \mathrm{~dB}$ at $2.4 \mathrm{GHz}$ and $5 \mathrm{GHz}$, respectively. To further improve the isolation, the device has the capability to provide the deep-OFF state by applying the voltage $\left(\mathrm{V}_{2}\right)$ between two electrodes of the deep-OFF state actuator. The movable electrodes move further away (switching from OFF state of $3 \mu \mathrm{m}$ to deepOFF state of $6 \mu \mathrm{m}$ ) from the signal lines to provide extra isolation compared to the initial isolation. In deep-OFF state, the isolations of $-22.39 \mathrm{~dB}$ at $2.4 \mathrm{GHz}$ and $-15.88 \mathrm{~dB}$ at $5 \mathrm{GHz}$ have been achieved.

The switch is micro-fabricated using MEMSCAP's SiliconOn-Insulator Process (SOIMUMPs), which offers a movable silicon structure layer of $25 \mu \mathrm{m}$ thickness. The SEM image of the fabricated device is shown in Fig. 3. To reduce the series resistance, a gold layer of $0.65 \mu \mathrm{m}$ is over-coated on the top and sidewall of the contactless switching areas by sputtering, as illustrated in the dashed area in Fig. 3.

\section{MeAsurement Results}

The RF performance of the fabricated contactless MEMS switch is characterized using RF Vector Network Analyzer (FieldFox ${ }^{\mathrm{TM}}$ N9923A) with ground-signal-ground (GSG) coplanar probes. The system is calibrated using the standard short-open-load-through on-wafer technique. The S-parameters of the input and output ports are extracted using the Vector Network Analyzer. The insertion loss and isolation of the switch are characterized by S21 from $1 \mathrm{GHz}$ to $6 \mathrm{GHz}$. The changes in insertion loss and isolation among different states are largely determined by the capacitance change resulting from the movement of the grounded electrodes. The capacitance is extracted from the S-parameters using the method provided in [5]. Fig. 4 illustrates the capacitance extracted from the measured S-parameters in comparison with the capacitance extracted from the CST-simulated S-parameters. It is found that the simulation results are in good agreement with the measured results. As shown in Fig. 4, the switch capacitance can be continuously tuned between the minimum and maximum values by adjusting the actuation voltages, $V_{1}$ and $V_{2}$. The measurement results show that the capacitance ranges from $60 \mathrm{fF}$ to $670 \mathrm{fF}$, which corresponds 


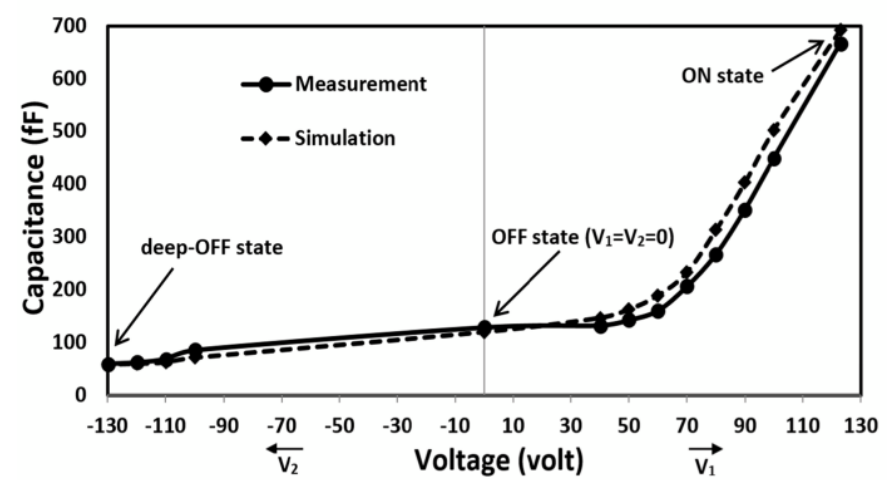

Fig. 4. Measured and simulated capacitances of the fabricated switch at various actuation voltages.

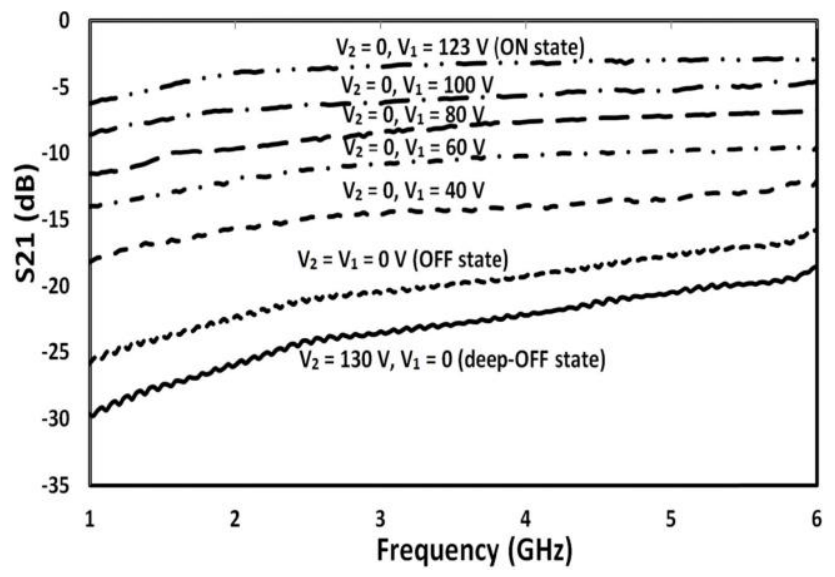

Fig. 5. Measured S21 parameter results of the fabricated switch at various actuation voltages.

to a maximum capacitance tuning ratio $\left(C_{\text {max }} / C_{\text {min }}\right)$ of 11.16 between deep-OFF state and $\mathrm{ON}$ state, as shown in Fig. 4. Similar tuning ratio (9.42) has been achieved using latching mechanisms [6].

In addition, the insertion loss and isolation of fabricated switch with various actuation voltages are shown in Fig. 5. In ON state, the insertion losses of $-3.62 \mathrm{~dB}$ at $2.4 \mathrm{GHz}$ and $-2.95 \mathrm{~dB}$ at $5 \mathrm{GHz}$ have been achieved with actuation voltage $\mathrm{V}_{1}$ of $123 \mathrm{~V}$. In OFF state $\left(\mathrm{V}_{1}=\mathrm{V}_{2}=0 \mathrm{~V}\right)$, the isolations are $-21.37 \mathrm{~dB}$ and $-17.7 \mathrm{~dB}$ at $2.4 \mathrm{GHz}$ and $5 \mathrm{GHz}$, respectively. In deep-OFF state $\left(\mathrm{V}_{1}=0 \mathrm{~V}\right.$, and $\left.\mathrm{V}_{2}=130 \mathrm{~V}\right)$, the isolations are $-24.43 \mathrm{~dB}$ and $-20.65 \mathrm{~dB}$ at $2.4 \mathrm{GHz}$ and $5 \mathrm{GHz}$, respectively. Compared to the OFF state, higher isolation has been achieved in the deep-OFF state with approximate $3 \mathrm{~dB}$ improvement across the full frequency range of $1 \mathrm{GHz}$ to $6 \mathrm{GHz}$. To further reduce the insertion loss in ON state, thicker metal overcoat layer can be applied to the contactless area if needed to reduce the series resistance [2], [7], [8].

\section{CONCLUSION}

In this letter, a continuously variable contactless RF MEMS switch is designed, fabricated and characterized for wireless application. The switch utilizes electrostatic actuation to tune the capacitance between the signal lines and movable electrodes fabricated inside the CPW. The switch has no physical contact between two electrodes, therefore, the stiction and charge injection issues occurred in traditional contact-type RF MEMS switches can be avoided. The isolation is further improved by actuating the device into the deep-OFF state. More than $20 \mathrm{~dB}$ difference has been achieved between $\mathrm{ON}$ and deep-OFF states, which is sufficient for RF switching applications in wireless frequencies. The insertion loss in ON state can be further improved by deposition of a thicker gold layer on the top and sidewall of switching electrodes by sputtering or evaporation process. Smaller gap (less than $0.5 \mu \mathrm{m})$ can also reduce the insertion loss. It is noticed that high actuation voltages were required in this design, however it can be reduced by designing more actuator electrodes or more flexible suspended springs. Future work includes the optimization of the design to improve the RF performance and reduce the applied voltage of the switch.

\section{REFERENCES}

[1] M. Barbato and G. Meneghesso, "A novel technique to alleviate the stiction phenomenon in radio frequency microelectromechanical switches," IEEE Electron Device Lett., vol. 36, no. 2, pp. 177-179, Feb. 2015. DOI: 10.1109/LED.2014.2376594

[2] J. Park, E. S. Shim, W. Choi, Y. Kim, Y. Kwon, and D.-I. Cho, "A non-contact-type RF MEMS switch for 24-GHz radar applications," J. Microelectromech. Syst., vol. 18, no. 1, pp. 163-173, Feb. 2009. DOI: 10.1109/JMEMS.2008.2011124

[3] M. Sterner, N. Roxhed, G. Stemme, and J. Oberhammer, "Static zeropower-consumption coplanar waveguide embedded DC-to-RF metalcontact MEMS switches in two-port and three-port configuration," IEEE Trans. Electron Devices, vol. 57, no. 7, pp. 1659-1669, Jul. 2010. DOI: 10.1109/TED.2010.2048239

[4] Y.-Q. Zhu, L. Han, L.-F. Wang, J.-Y. Tang, and Q.-A. Huang, "A novel three-state RF MEMS switch for ultrabroadband (DC-40 GHz) applications," IEEE Electron Device Lett., vol. 34, no. 8, pp. 1062-1064, Aug. 2013. DOI: 10.1109/LED.2013.2269993

[5] M.-H. Cho, G.-W. Huang, K.-M. Chen, and A.-S. Peng, "A novel cascadebased de-embedding method for on-wafer microwave characterization and automatic measurement," in IEEE MTT-S Int. Microw. Symp. Dig., Jun. 2004, pp. 1237-1240. DOI: 10.1109/MWSYM.2004.1339212

[6] D.-H. Baek, Y. Eun, D.-S. Kwon, M.-O. Kim, T. Chung, and J. Kim, "Widely tunable variable capacitor with switching and latching mechanisms," IEEE Electron Device Lett., vol. 36, no. 2, pp. 186-188, Feb. 2015. DOI: 10.1109/LED.2014.2378272

[7] M. Sterner, N. Roxhed, G. Stemme, and J. Oberhammer, "Electrochemically assisted maskless selective removal of metal layers for three-dimensional micromachined SOI RF MEMS transmission lines and devices," J. Microelectromech. Syst., vol. 20, no. 4, pp. 899-908, Aug. 2011. DOI: 10.1109/JMEMS.2011.2159100

[8] A. Q. Liu, M. Tang, A. Agarwal, and A. Alphones, "Lowloss lateral micromachined switches for high frequency applications," J. Micromech. Microeng., vol. 15, no. 1, pp. 157-167, 2005. DOI: $10.1088 / 0960-1317 / 15 / 1 / 023$ 